\title{
Research on the design of emergency services for nursing homes
}

\author{
Jiawei Liu ${ }^{1}$, Hao Qian ${ }^{2}$ and Dongming $\mathrm{Ma}^{1 *}$ \\ ${ }^{1}$ Departments of Mechanical and Electrical Engineering, University of Beijing University of Chemical Technology, 100029, china \\ ${ }^{2}$ Departments of Mechanical and Electrical Engineering, University of Beijing University of Chemical Technology, 100029, china
}

\begin{abstract}
In order to improve the efficiency of medical treatment for the elderly during sudden illness and optimize the first-aid service process in nursing homes, this paper uses the SET analysis method to obtain the design gap of the existing nursing-home emergency service system. Taking the elderly with coronary heart disease as an example, focus on their service acceptance and healthy lifestyle. Use the method of user journey map to visualize the daily behavior of the elderly, followed by the SWOT analysis method to obtain the market competitive advantage, and finally establish a coronary heart disease first-aid service system for nursing homes. It is hoped that this study will provide emergency medical service plans for elderly patients with sudden acute illness and provide a reference for the emergency treatment process of other types of diseases.
\end{abstract}

\section{Introduction}

The death rate of cardiovascular disease in the elderly is much higher than that of cancer. Every year, more than 1 million elderly people die because of sudden illness and they cannot receive timely treatment. Elderly people often panic when they encounter sudden illness without being accompanied. At present, there is a problem of unresponsiveness and irregular service in the emergency system of nursing homes. First aid is more passive. Through the perfect first aid support service process, it can effectively reduce the first aid steps for elderly patients with heart attacks, improve the efficiency of medical treatment, and bring a positive impact on society.

\section{Background analysis}

\subsection{Trend analysis of acute diseases in the elderly}

According to China's aging population data, in 2018, the population over 65 years old was $1,665.8$ billion, and the proportion of the population over 65 years old accounted for $11.9 \%$. Nearly $90 \%$ of the sudden deaths caused by elderly diseases are due to heart [1]. The survey shows that the popularization rate of first aid knowledge is very low in the country. In an emergency, $98 \%$ of people only call the emergency call to contact the medical staff, and did not carry out other rescue measures, so that they missed the opportunity for rescue.

\subsection{Nursing home emergency service system based on SET mode}

At present, the aging trend in China is severe, and the first aid service process in nursing homes is not yet perfect. Using SET analysis, comprehensive analysis of social trends, economic power, and advanced technology. In this way, the background and direction of the design of the emergency aid system for nursing homes were further clarified [2].

(1) Society: With the change of the social population structure, especially the adjustment of the urban population structure, the phenomenon of "aging" has become a hot topic in the society that the public is concerned about. In the field of elderly care, China can be said to have had preliminary development, but the development of emergency care is almost blank [3].

(2) Economy: The China Aging Industry Development Report issued by the National Working Committee on Aging, the consumption potential of China's elderly population will increase from 4 trillion to 106 trillion yuan between 2014 and 2050. The two-way development of the pension industry and the "silver economy" not only brings new solutions to the problem of old-age care, but also injects new impetus into the high-quality development of the economy.

(3) Technology: After consulting relevant literature, it is found that most of the new pension models and medical services are based on Internet technology and information technology, which can be subdivided into the Internet of Things, cloud computing, intelligent perception technology, and emotional interaction. During the COVID-19 outbreak, the Internet and information

"Corresponding author's e-mail: 99308430@qq.com 
technology have played an active role in epidemic prevention and national health care, and have been highly recognized by relevant national departments, society and the public.

After the above analysis, the following design directions are obtained: starting from solving the health crisis of the elderly brought by social aging, focusing on emergencies caused by elderly diseases, and providing emergency medical services based on smart technology.

\section{Service design concept}

\subsection{Service design}

We can define service design as: taking users as the center, in order to enhance user experience, systematically construct, design and reorganize tangible and intangible contact points in services, and bring added value to products and services. As a process of service design, it is driven by design thinking and finds creative solutions for users or businesses through research analysis and iterative development [4].

\subsection{Service design applied to first aid medical products}

The demand for elderly health services of the elderly in our country is growing rapidly with high quality and diversification. In addition, according to the estimate of the market demand for geriatric medical services, the demand for geriatric medical services in China will reach 669.7 billion yuan by 2024 . This article aims to establish a medical system for elderly emergency services, using user-centered service design methods to achieve the accurate implementation and scientific management of emergency procedures.

\section{Design of emergency medical aid services for nursing homes--Taking elderly patients with coronary heart disease as an example}

\subsection{Target user analysis}

In order to make the research more targeted and reasonable, and the elderly are currently facing many diseases, taking the elderly with coronary heart disease as an example, 100 target users in 3 target nursing homes were selected for research. The author summarizes its service acceptance, first aid psychology and healthy living situation:

(1) The elderly actively face the risks brought by the disease, maintain an optimistic attitude towards intelligent medical and technological services, and are willing to experience efficient and intelligent service management methods.

(2) Once an elderly person with coronary heart disease becomes ill without a guardian, he will have a physical condition with an increased heart rate and increased blood pressure, and at the same time be accompanied by a nervous sense of pressure, leading to a decline in the elderly ability to save themselves.

(3) Forgetfulness, the elderly generally put medicine bottles of quick-acting rescue pills or nitroglycerin in the pockets of clothes. It is easy to forget to take the medicine bottles after changing clothes.

\subsection{User journey graph analysis}

User journey diagram is one of the important tools in the service design exploration stage, which can intuitively and systematically show the user's behavior to the researchers. As shown in Figure 1, the author divides the behavior of the elderly with coronary heart disease into six stages. After decomposing the user's behavior, the user's pain points and sweet points are found, the emotion curve is drawn, and the product design point is found through the visualization curve. Among them, the pain points of the elderly behavior when they go out and forget to take medicine, unattended heart attack, and the inconvenience of taking medicine are at the lowest point of emotion. From these directions, we will start to improve the first aid service process for elderly patients with sudden heart disease. 


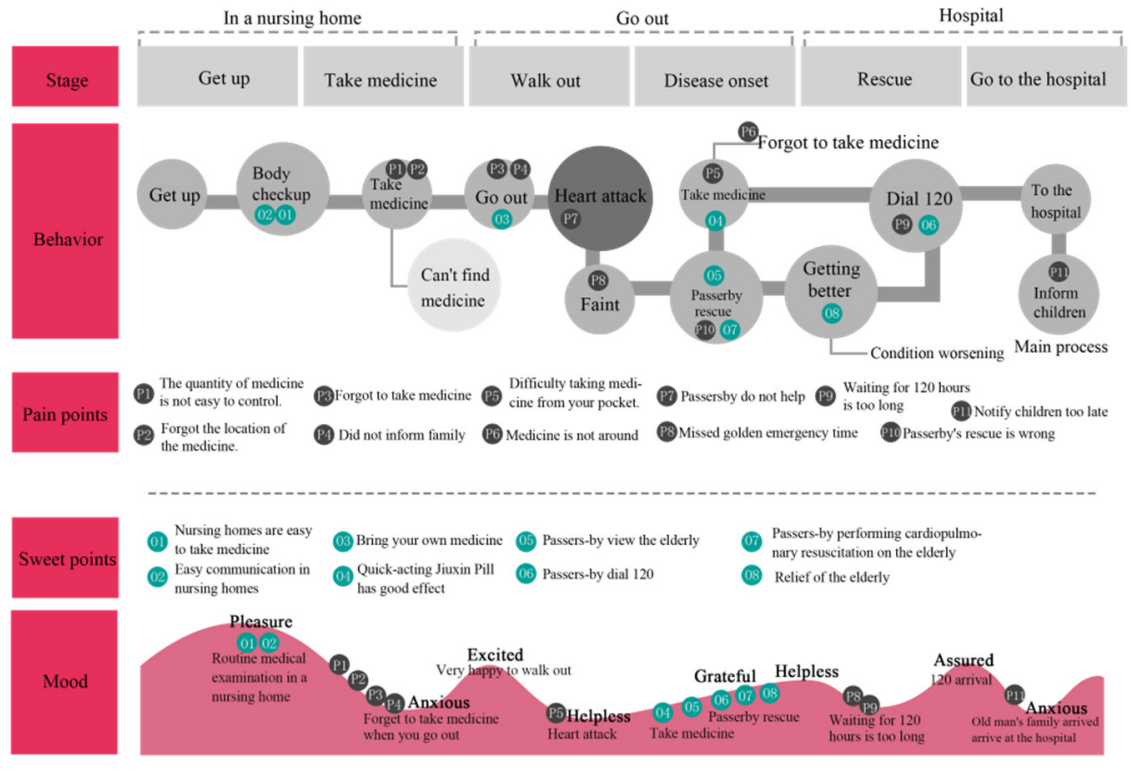

Figure 1. User journey map

\subsection{SWOT analysis}

The SWOT analysis method can integrate the company's own advantages, disadvantages and opportunities and threats in external competition, and is of great significance for formulating excellent development strategies. As can be seen from Figure 2 below, the market competitive advantages of the emergency care system for the elderly can be formulated as follows: (1)

Strenghts
1、Mass establishment of
older communities
2、 Diversified development
of technology
3、 Wide application of ser-
vice design
Opportunities
1、Elderly emergency care
products are blank
2、The prosperity of the
"silver economy"
3、The elderly has a large
population

Promote the development of intelligent nursing homes, embedding multidimensional integration of intelligent technology and elderly care services. (2) Starting from the life and education background of the elderly, develop medical technology products with strong applicability. (3) A multifaceted and systematic study of the silver hair economy, used to guide the design of services and products.

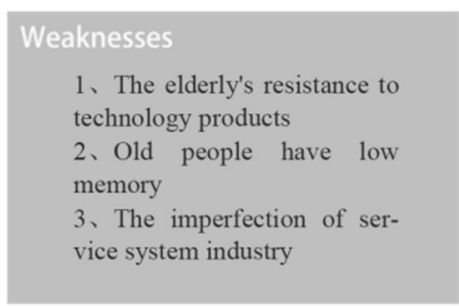

Threats

$$
\begin{aligned}
& \text { 1、 There are many organiza- } \\
& \text { tions to defraud the elderly } \\
& \text { 2、The proliferation of tech- } \\
& \text { nology products }
\end{aligned}
$$

Figure 2. SWOT analysis

\subsection{Service system positioning}

As shown in Figure 3, after the above preliminary investigation and comprehensive analysis, the positioning of the service system is obtained (1) caring, symbolizing first aid medical products. (2) Nationality, improve national understanding of coronary heart disease first aid, and reduce sudden cardiac death rate. (3) Sociability, establish a first-aid service system for nursing homes, and increase social attention. 


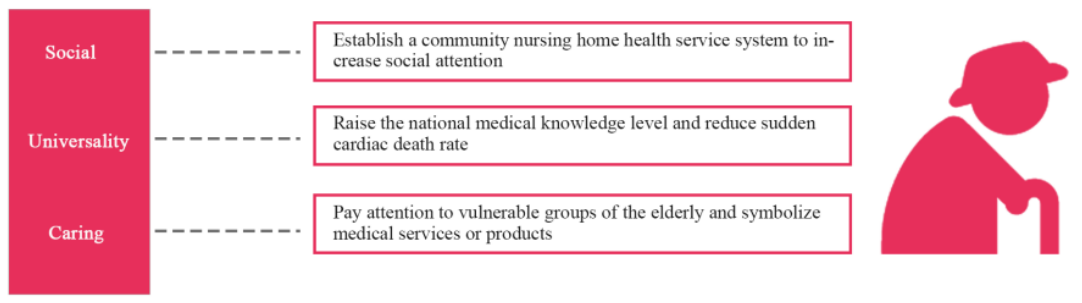

Figure 3. Service system positioning

\section{Map of first aid medical service system}

\subsection{Smart wearable bracelet design}

In order to meet the information transmission between the elderly and nursing homes, hospitals, etc., the development of nursing home emergency service system needs to carry out synchronous design on hardware

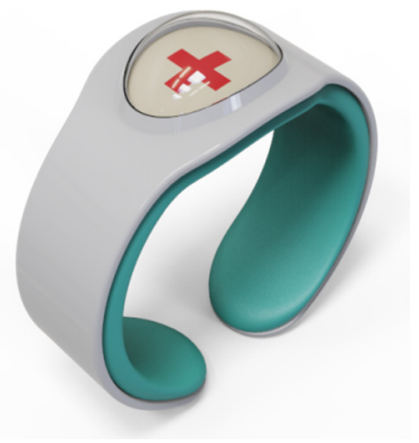

products [5]. Through user analysis and SET research, the smart wearable bracelet should realize the following functional classification: monitoring the heart rate, blood pressure and other signs of the elderly. Positioning and reminding medication function. It is connected with the equipment in the nursing home to realize the storage and transmission of information. The specific shape is shown in Figure 4.

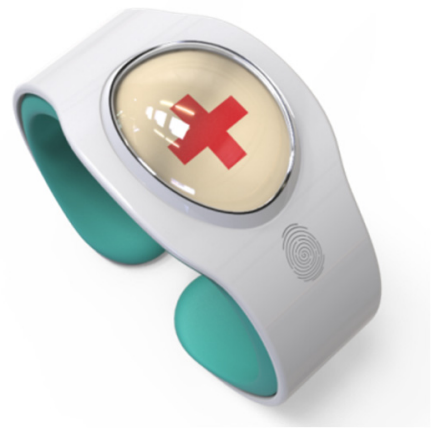

Figure 4. Bracelet shape

\subsection{Service system design}

Through the analysis of the macro-environment of the first-aid pension market and target users, the following modules for the construction of the first-aid service system are obtained: (1) Regional services. In the elder care community, through the first aid bracelet to the elderly body first aid signal, quickly transmitted back to the nursing home and medical station, to achieve seamless rescue, seize the golden rescue time. (2)
Continuous monitoring of signs information. When the elderly wear the bracelet to go out, they can transmit the body information to the user's family members, nursing homes and medical stations anytime and anywhere, so as to realize the all-day protection of the patients. (3) Establish a virtual disease communication platform. The communication between the old man and friends through the app matching the bracelet eliminates the old man's sense of loneliness and fear of illness. As shown in Figure 5. 


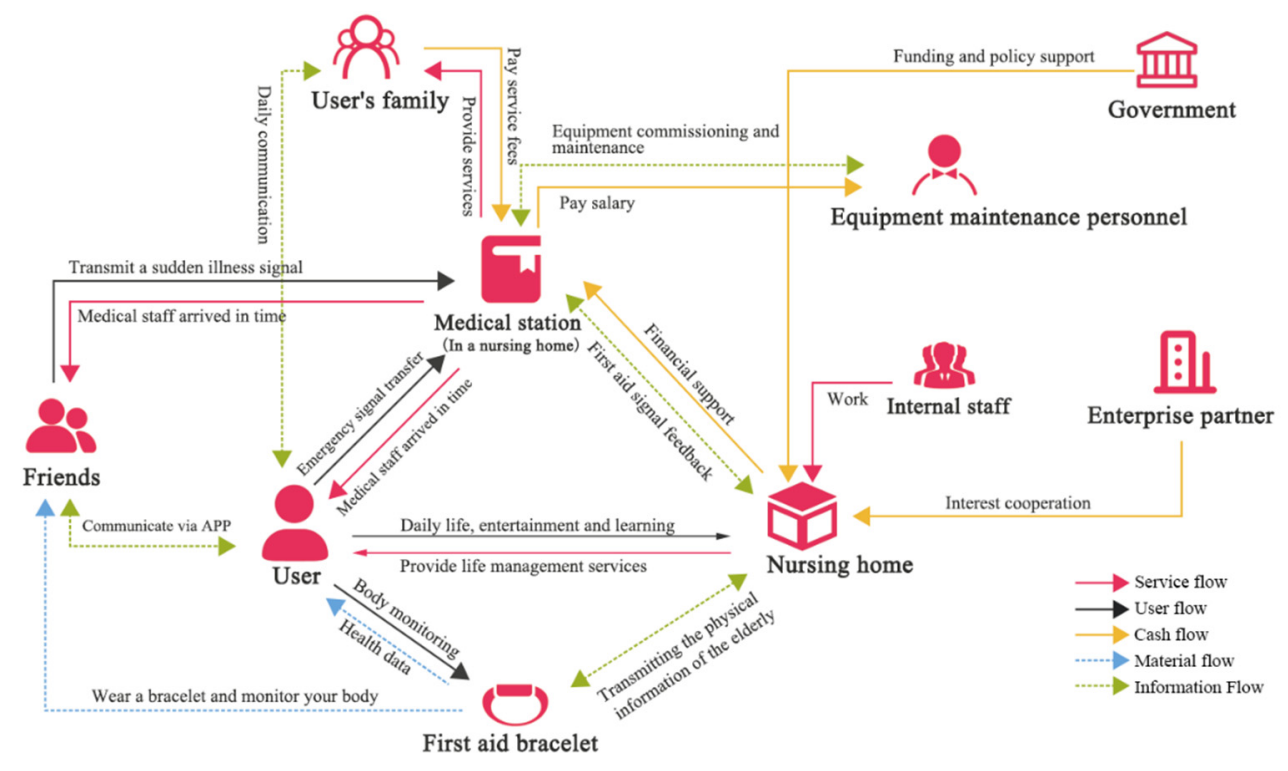

Figure 5. Service system

\section{Conclusion}

The design of the first-aid system for the sudden illness of the elderly, using the service design method, through SET analysis and target user surveys of the elderly first-aid industry, we obtained the combination of elderly community services and medical products based on the needs of the elderly service system. Realized full-time disease monitoring and personalized emergency services for the elderly, optimized the user experience process, and provided reference ideas for the elderly emergency medical market.

\section{References}

1. Li Zhan-fa, Tan Li-hui. The effect of pre-hospital emergency treatment on acute myocardial infarction[J]. China Urban and Rural Enterprise Health, 2019, 34 (11): 65-66.

2. Cao En-guo, Zhang Xin, Deng Rong, Zhao Ya-peng, Li Shi-guo, Zhang Han-ning. Research on the design of home-based pension interactive product system based on SET analysis [J]. Mechanical Design, 2014, 31 (12): 119-122.

3. Arterial Network. (2018) In-home nursing homes can save up to $90 \%$ of their costs by using telemedicine bedside care.https:/vcbeat.top/.

4. Zhang chang-juan. Research on the design of health management services for nursing homes [D]. Jiangnan University, 2017.

5. Wang Yi, Zhao Gang. Interpretation of quasi-physical design of mobile interactive interface [J]. Packaging Engineering, 2013 (18): 58-64. 